PROCEEDINGS OF THE AMERICAN MATHEMATICAL SOCIETY

Volume 126, Number 3, March 1998, Pages 867-876

S 0002-9939(98)04103-3

\title{
WEAK CHOICE PRINCIPLES
}

\author{
OMAR DE LA CRUZ AND CARLOS AUGUSTO DI PRISCO
}

(Communicated by Andreas R. Blass)

\begin{abstract}
We consider several weak forms of the Axiom of Choice obtained debilitating some well known choice principles. Implication relations among them and independence results are established using, in some cases, permutation models or symmetric models. The relationship between Ramsey's Theorem and these choice principles is also explored, showing that, in this context, the known results are the best possible.
\end{abstract}

\section{INTRODUCTION}

In this article we will consider several forms of choice obtained by debilitating in certain specific ways some well known choice principles. In some cases the debilitating procedures give rise to already known principles, in other cases new choice principles are obtained.

The choice principles we will be dealing with are the following, SELECTION PrinCiple (SP), Ordering Principle (OP), Axiom of Choice for Well Orderable Sets (ACWO), Axiom of Choice for Finite Sets (ACF), Axiom of Choice For Finite sets of $n$ ELEMENTs $\left(\mathbf{C}_{n}\right)$.

The implications in the next theorem are well known, and none of them can be reversed. Also, ACWO does not imply OP. Proofs can be found, for example, in [Je1]. In [Pi2] it is shown that SP does not imply ACWO.

\section{Theorem 1.1.}

$$
\begin{gathered}
\mathrm{AC} \Rightarrow \mathbf{S P} \Rightarrow \mathbf{O P} \Rightarrow \mathbf{A C F} \Rightarrow \forall n \mathbf{C}_{n} \Rightarrow \mathbf{C}_{m} \\
\mathbf{A C} \Rightarrow \mathbf{A C W O} \Rightarrow \mathbf{A C F} .
\end{gathered}
$$

The Axiom of Choice (AC) can be expressed as " $\forall X$ there is a choice function for the collection of non-empty subsets of $X$ ". We can debilitate this statement by requiring the choice function to exist only on the collection of non-empty subsets of an infinite subset of $X$, obtaining "For every infinite $X$ there is an infinite $Y \subseteq X$ and a choice function for the collection of non-empty subsets of $Y$ ", which is called the restricted version of $\mathbf{A C}$ and is denoted by $\mathbf{A} \mathbf{C}^{\diamond}$. Observe that $\mathbf{A} \mathbf{C}^{\diamond}$ is equivalent to "Every infinite set contains a countable subset".

Received by the editors February 28, 1996 and, in revised form, August 30, 1996.

1991 Mathematics Subject Classification. Primary 03E25, 04 A25.

The authors thank Carlos Gonzalez and David Pincus for conversations on the subject of this article. 
Besides $\mathbf{A} \mathbf{C}^{\diamond}$, we will consider the following "restricted" choice principles:

$\mathbf{A C F}^{\diamond}$ : For every infinite set $X$ there exists $Y \subseteq X, Y$ infinite, and a choice function for the collection of finite non-empty subsets of $Y$;

$\mathbf{S P}^{\diamond}$ : For every infinite set $X$ there is an infinite subset $Y$ and a function assigning a proper non-empty subset to every subset of $Y$ with at least two elements;

$\mathbf{O P}^{\diamond}$ : For every infinite set $X$ there is an infinite subset $Y$ and a linear order for $Y$;

$\mathbf{A C W O}^{\diamond}$ : For every infinite set $X$ there is an infinite subset $Y$ and a function assigning one of its elements to every non-empty well orderable subset of $Y$;

$\mathbf{C}_{n} \diamond$ : For every infinite set $X$ there is an infinite subset $Y$ and a function choosing one element from each $n$-element subset of $Y$.

$\mathbf{A C W O}^{\diamond}$ and $\mathbf{A C F} \mathbf{C F}^{\diamond}$ turn out to be equivalent. One implication is obvious; for the other, for $X$ infinite, if it has a countable infinite subset $Y$, then an enumeration of $Y$ provides a well order for every non-empty subset of $Y$ (and therefore a choice function for the collection of such subsets of $Y$ ); if there is no countable infinite subset $Y$ of $X$, then the only well orderable subsets of $X$ are the finite ones.

The restricted version of a choice principle depends on the specific way the original principle is stated. Restricted versions of two equivalent choice principles might give non-equivalent principles. So, we take the list above as the definitions of our restricted choice principles.

We define now another set of choice principles. Choice principles are frequently stated postulating the existence of a choice function for every family of sets in a certain class, $\mathrm{P}=(\forall F \in \mathcal{S})$ ( there is a selector for $F$ ), where $\mathcal{S}$ is a class of nonempty sets.

These principles can be weakened as follows to obtain what we will call their diminished versions: $\mathrm{P}^{-}=(\forall F \in \mathcal{S}$ infinite $)\left(\exists F^{\prime} \subset F\right.$ infinite $)($ there is a selector for $\left.F^{\prime}\right)$.

If we state $\mathbf{A C}$ as "For every family of non-empty sets there is a choice function", then $\mathbf{A} \mathbf{C}^{-}$is "For every infinite family $\mathrm{F}$ of non-empty sets, there is an infinite subfamily with a choice function".

Notice that this is different from the restricted version stated above; in fact, this diminished version of $\mathbf{A C}$ is equivalent to $\mathbf{A C}_{\omega}$ (see $[\mathrm{Br}]$ ).

Other "diminished" principles we will consider are:

$\mathbf{S P}^{-}$: For every infinite family $F$ of non-empty sets with at least two elements each, there is an infinite subfamily $F^{\prime} \subset F$ and a function assigning a proper subset to each element of $F^{\prime}$.

$\mathbf{A C W O}^{-}$: For every infinite family $F$ of non-empty well orderable sets, there is an infinite subfamily $F^{\prime} \subset F$ with a choice function.

$\mathbf{A C F}^{-}$: For every infinite family $F$ of finite non-empty sets, there is an infinite subfamily $F^{\prime} \subset F$ with a choice function.

$\mathbf{C}_{n}{ }^{-}$: For every infinite family $F$ of finite non-empty sets of $n$ elements, there is an infinite subfamily $F^{\prime} \subset F$ with a choice function.

Each diminished or restricted principle follows from the corresponding choice principle. The implications are obvious, and the fact that they are strict can be shown using permutation models with countable support, as will be indicated at the end of Section 2.

All the implications in Theorem 1.1 remain true when each principle is replaced by its restricted version. For the diminished principles, an interesting point is worth mentioning. Since $\mathbf{O P}$ does not have a diminished version, it is not clear if $\mathbf{S P}^{-}$ 
implies $\mathbf{A} \mathbf{C F}{ }^{-}$. The rest of the implications of Theorem 1.1 remain true for the diminished principles.

The relationship between restricted and diminished versions is interesting since implications cannot be established in the same way for all the choice principles. For $\mathbf{A C}$, the diminished version strictly implies the restricted version (i.e. $\mathbf{A C}_{\omega}$ strictly implies "every infinte set contains a countable subset"), whereas for ACF a strict implication goes in the opposite direction. For ACWO and for SP neither implication holds.

Theorem 1.2. (1) $\mathbf{A C}^{-}$strictly implies $\mathbf{A C}^{\diamond}$.

(2) $\mathbf{A C F} \mathbf{C F}^{\diamond}$ strictly implies $\mathbf{A C F ^ { - }}$.

(3) $\mathbf{A C W O}^{\diamond}$ neither implies nor is implied by $\mathbf{A C W O}^{-}$.

(4) $\mathbf{S P}^{\diamond}$ neither implies nor is implied by $\mathbf{S P}^{-}$.

This theorem will be proved in Section 3. In Section 2 a permutation model is presented; Section 4 contains some remarks about the relationship between Ramsey's Theorem and the weak choice principles considered. In particular we will see that the known implications between Ramsey's Theorem and choice principles are optimal in a certain sense. The relationship between $\mathbf{C}_{n} \diamond$ and $\mathbf{C}_{n}{ }^{-}$is not fully understood. It is easy to show that for $n=2,3, \mathbf{C}_{n} \diamond$ strictly implies $\mathbf{C}_{n}{ }^{-}$. Montenegro [Mo] showed that the implication is true for $n=4$, but nothing is known for $n \geq 5$. Note that for every $n \in \omega, \mathbf{C}_{n}{ }^{-}$holds in the basic Fraenkel Model but $\mathbf{C}_{n} \diamond$ does not. It is obvious that $\left(\forall n \mathbf{C}_{n}\right)^{\diamond}$ implies $\left(\forall n \mathbf{C}_{n} \diamond\right)$, but it is not clear if this implication is strict.

\section{A permutation model}

For the theory of permutation models and symmetric models as well as for notation we refer the reader to [Je1] or [Je2].

Permutation models will only give us independence results in the Set Theory with Atoms (ZFA); corresponding independence results are obtained for ZF invoking transfer theorems of Jech-Sochor [Je1] and Pincus [Pi1].

We describe now a permutation model, the Cylinder Model, in which the set of atoms is organized in a countable collection of countable dense "circles" which can rotate rigidly.

More formally; we consider the additive group $R^{1}=\mathbb{Q} / \mathbb{Z}$ (isomorphic to the subgroup of $S^{1}$ formed by the points with rational argument). To define the group $\mathcal{G}$ of permutations consider $A$ organized as $A=\left\{a_{i}^{p}: i \in \omega, p \in R^{1}\right\}$, where $a_{i}^{p}=a_{j}^{q}$ implies $p=q$ and $i=j$. $A$ is thus the union of a countable collection of copies of $R^{1}$; we define $A_{i}=\left\{a_{i}^{p}: p \in R^{1}\right\}$, for each $i \in \omega$.

Define $\mathcal{G}$ as the group generated by $\left\{\pi_{i}^{p}: i \in \omega, p \in R^{1}\right\}$, where $\pi_{i}^{p}\left(a_{i}^{q}\right)=a_{i}^{p+q}$ and $\pi_{i}^{p}\left(a_{j}^{q}\right)=a_{j}^{q}$ if $j \neq i$.

The Cylinder Model (CM) is obtained using this group and the ideal $\mathcal{I}$ of finite supports.

Lemma 2.1. For each $n \geq 2$, the family $[A]^{n}$ does not have a choice function in CM.

Proof. $[A]^{n}$ is clearly in the model. Suppose that $f$ is a selector for the family of $n$-element sets of atoms, and let $E$ be a support for $f$. Let $k \in \omega$ be such that $A_{k} \cap E=0$; consider the permutation $\pi=\pi_{k}^{[1 / n]}$, where $[r]$ is the coset 
corresponding to the rational $r$. If $Y=\left\{a_{k}^{[0]}, a_{k}^{[1 / n]}, a_{k}^{[2 / n]}, \ldots, a_{k}^{[n-1 / n]}\right\}$ ( $Y$ is the set of vertices of a regular polygon of $n$ vertices), then,

$$
\begin{aligned}
\pi Y & =\pi\left(\left\{a_{k}^{[0]}, a_{k}^{[1 / n]}, a_{k}^{[2 / n]}, \ldots, a_{k}^{[(n-1) / n]}\right\}\right) \\
& =\left\{a_{k}^{[0+(1 / n)]}, a_{k}^{[(1 / n)+(1 / n)]}, a_{k}^{[(2 / n)+(1 / n)]}, \ldots, a_{k}^{[(n-1) / n+(1 / n)]}\right\} \\
& =\left\{a_{k}^{[1 / n]}, a_{k}^{[2 / n]}, a_{k}^{[3 / n]}, \ldots, a_{k}^{[0]}\right\} \\
& =Y
\end{aligned}
$$

and $\pi \in \operatorname{fix}(E) \subset \operatorname{sym}(f)$. So, $(Y, f(Y)) \in f$ implies $\pi(Y, f(Y)) \in \pi f$. It follows that $(Y, \pi(f(Y))) \in f$, since $\pi \in \operatorname{sym}(Y)$ and $\pi \in \operatorname{sym}(f)$. But $f$ is a function, so $\pi(f(Y))=f(Y) ; f$ being a choice function, $f(Y) \in Y$, but no element of $Y$ is fixed under $\pi$. Contradiction.

Lemma 2.2. CM satisfies "Every infinite set contains a countable subset".

Proof. The proof follows ideas of [Bl]. Recall that in a permutation model, a supersupport for a set $X$ is a support for $X$ which also supports each element of $X$. For $X \in \mathrm{CM}$ infinite, we need only consider the case in which $X$ is not well orderable. By [Je1, (4.2), p. 47] there is no finite supersupport $E$ for $X$. So if we fix a support $E_{0}$ for $X$, we have that $\operatorname{fix}(X) \not \supset$ fix $\left(E_{0}\right)$, and therefore there are $\pi \in \operatorname{fix}\left(E_{0}\right)$ and $x_{0} \in X$ such that $\pi \notin \operatorname{sym}\left(x_{0}\right)$. Fix also such $x_{0}$, and observe that $\operatorname{sym}\left(x_{0}\right) \not \supset$ fix $\left(E_{0}\right)$. If $E$ is a support for $x_{0}$, then, $E \not \subset E_{0}$. We pick a support $E_{1}$ for $x_{0}$ such that $E_{1} \backslash E_{0}$ has the least possible number of elements. Fix $a \in E_{1} \backslash E_{0}$, and let $F=E_{1} \backslash\{a\}$. Let $k$ be such that $a \in A_{k}$, and define now a function $f: A_{k} \rightarrow X$ by $f=\left\{\left(\pi a, \pi x_{0}\right): \pi \in \operatorname{fix}\left(F \cup E_{0}\right)\right\}$.

As in [Bl], $f$ is a function in CM, its domain is $A_{k}$ and $f^{\prime \prime} A_{k} \subset X$.

To complete the proof we only need to show that $f^{\prime \prime} A_{k}$ is infinite.

Let $h: R^{1} \rightarrow \operatorname{fix}\left(F \cup E_{0}\right)$ be defined by $h(p)=\pi_{k}^{p}$. range $(h) \subset \operatorname{fix}\left(F \cup E_{0}\right)$, since $\left(F \cup E_{0}\right) \cap A_{k}=0$. Clearly $h$ is a monomorphism, and $G=\left\{p \in R^{1}: h(p)\left(x_{0}\right)=x_{0}\right\}$ is a subgroup of $R^{1}$. Each element of $R^{1} / G$ gives rise to a different element of $f^{\prime \prime} A_{k}$, since if $p, q \in R^{1}$ are such that $p-q \notin G$, then $h(p-q)\left(x_{0}\right) \neq x_{0}$, that is to say,

$$
\begin{aligned}
h(q)^{-1} h(p)\left(x_{0}\right) & \neq x_{0}, \\
h(p)\left(x_{0}\right) & \neq h(q)\left(x_{0}\right), \\
\pi_{k}^{p} x_{0} & \neq \pi_{k}^{q} x_{0}, \\
f\left(\pi_{k}^{p} a_{0}\right) & \neq f\left(\pi_{k}^{q} a_{0}\right) .
\end{aligned}
$$

We also have that $R^{1} \neq G$, since otherwise we contradict the minimality of $E_{1}$. So the following easy lemma suffices to get that $f^{\prime \prime} A_{k}$ is infinite.

Lemma 2.3. There is no proper subgroup $G$ of $R^{1}$ such that $R^{1} / G$ is finite.

So, we have $f: A_{k} \rightarrow X$ with $f^{\prime \prime} A_{k}$ infinite, and $A_{k}$ countable (in fact, $\operatorname{fix}\left(A_{k}\right) \supset$ fix $(\{a\})$, therefore $\{a\}$ supports the bijection $p \mapsto a_{k}^{p}$ from $R^{1}$ onto $A_{k}$, since $\pi \in$ fix $(\{a\})$ and $\pi\left(p, a_{k}^{p}\right)=\left(\pi p, \pi a_{k}^{p}\right)=\left(p, a_{k}^{p}\right)$ since $p$ is in the kernel of CM).

Given a bijection $g: \omega \rightarrow A_{k}$, it is easy to find a countable infinite subset of $X$.

Theorem 2.4. $\mathbf{A} \mathbf{C}^{\diamond}$ does not imply $\mathbf{C}_{n}$ for any $n \geq 2$.

Proof. It follows from the previous lemmas that $\mathrm{CM} \models\left(\mathbf{A} \mathbf{C}^{\diamond}+\neg \mathbf{C}_{n}\right)$. 
Theorem 2.4 also follows from results of [Pi2], but the proof given here is simpler. D. Pincus constructed a model where $\mathbf{A} \mathbf{C}^{\diamond}$ holds and there is a countable family of countable sets without a choice function ([Pi]; see also [Je1], p. 132, problem 4); this is also the case in the cylinder model. Pincus' model is also a permutation model with a countable collection of countable sets of atoms, the group of permutations being the group generated by the permutations of each of these sets.

From Theorem 2.4 it follows that none of the restricted versions of the choice principles we have considered implies its original version. This is so because all the restricted versions, $\mathbf{S P}^{\diamond}, \mathbf{O} \mathbf{P}^{\diamond}, \mathbf{A} \mathbf{C F} \mathbf{F}^{\diamond}$, and each $\mathbf{C}_{m} \diamond$, are implied by $\mathbf{A} \mathbf{C}^{\diamond}$, but each of the original versions implies some $\mathbf{C}_{n}$.

Observe also that $\mathbf{A C W O}^{-}$does not hold in $\mathrm{CM}$, since the collection $\left\{A_{i}\right.$ : $i \in \omega\}$ of well ordered sets does not have an infinite subcollection with a selector. Therefore $\mathbf{A} \mathbf{C}^{-}$is also false in $\mathrm{CM}$ and so neither of these diminished principles is deducible from $\mathbf{A C}^{\diamond}$.

Ramsey's Theorem holds in this model, since it is a consequence of $\mathbf{A C} \mathbf{C}^{\diamond}$. $\mathbf{A C F} \mathbf{C F}^{-}$ and its consequences, $\forall n \mathbf{C}_{n}{ }^{-}$and each of the $\mathbf{C}_{m}{ }^{-}$, follow from $\mathbf{A C F}{ }^{\diamond}$ as we will see in the next section, and so, also hold in CM.

A way to see that each of the choice principles implies strictly its reduced and diminished versions is to build a permutation model from a set $A$ of atoms of cardinality $\aleph_{1}$, taking the group of all the permutations and the ideal of countable supports. In the model thus obtained none of the principles $\mathbf{C}_{n}$ hold. It is enough to notice that the family $[A]^{n}$ is in the model but has no choice function. In consequence, none of the principles $\mathbf{A C}, \mathbf{S P}, \mathbf{O P}, \mathbf{A C F}$ holds in this model. Nevertheless, $\mathbf{A C}^{-}$holds here. In fact, let $F$ be an infinite collection of non-empty sets. Take in the base model a countable subcollection $F^{\prime}$ and a choice function $f$ for it (the base model satisfies ZFA+AC). Pick a support $E_{n}$ for each $X_{n} \in F^{\prime}$, and a support $E_{n}^{\prime}$ for each $f\left(X_{n}\right)$. The union $E=\bigcup_{n \in \omega} E_{n} \cup \bigcup_{n \in \omega} E_{n}^{\prime}$ is countable and it is a support for $f$.

\section{Proof of Theorem 1.2}

Part 1 of Theorem 1.2 is well known. The fact that $\mathbf{A \mathbf { C } ^ { \diamond }}$ does not imply $\mathbf{A C}^{-}$ can be obtained using the cylinder model where $\mathbf{A} \mathbf{C}^{\diamond}$ holds but $\mathbf{A C}^{-}$does not.

Lemma 3.1. $\mathbf{A C F}^{\diamond}$ implies $\mathbf{A C F}^{-}$.

Proof. This implication is easy. Given a family $F$ of pairwise disjoint finite sets, $\mathbf{A C F}^{\diamond}$ implies that there is an infinite $Y \subset \bigcup F$ with a choice function $f$ defined on the set of its finite subsets. Clearly $G=\{a \in F: a \cap Y \neq 0\}$ is infinite, and so we define a choice function on $G$ as follows: $g(a)=f(a \cap Y)$ for all $a \in G$.

In [Bl] it is shown that Ramsey's Theorem holds in the Basic Fraenkel Model (BFM), and this implies that $\mathbf{A C F}^{-}$holds there (see [Kl2]). On the other hand, in this model the set of atoms is amorphous; see [Je1, p. 52]. Truss proved in [Tr] that for any $n>1, \mathbf{C}_{n}$ implies that there are no amorphous sets, the same proof works for $\mathbf{C}_{n} \diamond$, and therefore $\mathbf{C}_{n} \diamond$ is not true in this model for any $n$ (nor $\mathbf{A} \mathbf{C F}{ }^{\diamond}$, in consequence). In conclusion, Fraenkel's Basic Model provides a model of ZFA where $\mathbf{A} \mathbf{C F}^{-}$holds but $\mathbf{A C F} \mathbf{C F}^{\diamond}$ does not.

Next we will prove that $\mathbf{A C W O}^{-}$holds in the Basic Fraenkel Model. ACWO $\mathbf{A C}$ does not hold there (since it implies that there are no amorphous sets). We will also show that in this model $\mathbf{S P}^{-}$holds and $\mathbf{A} \mathbf{C}^{-}$is false. 


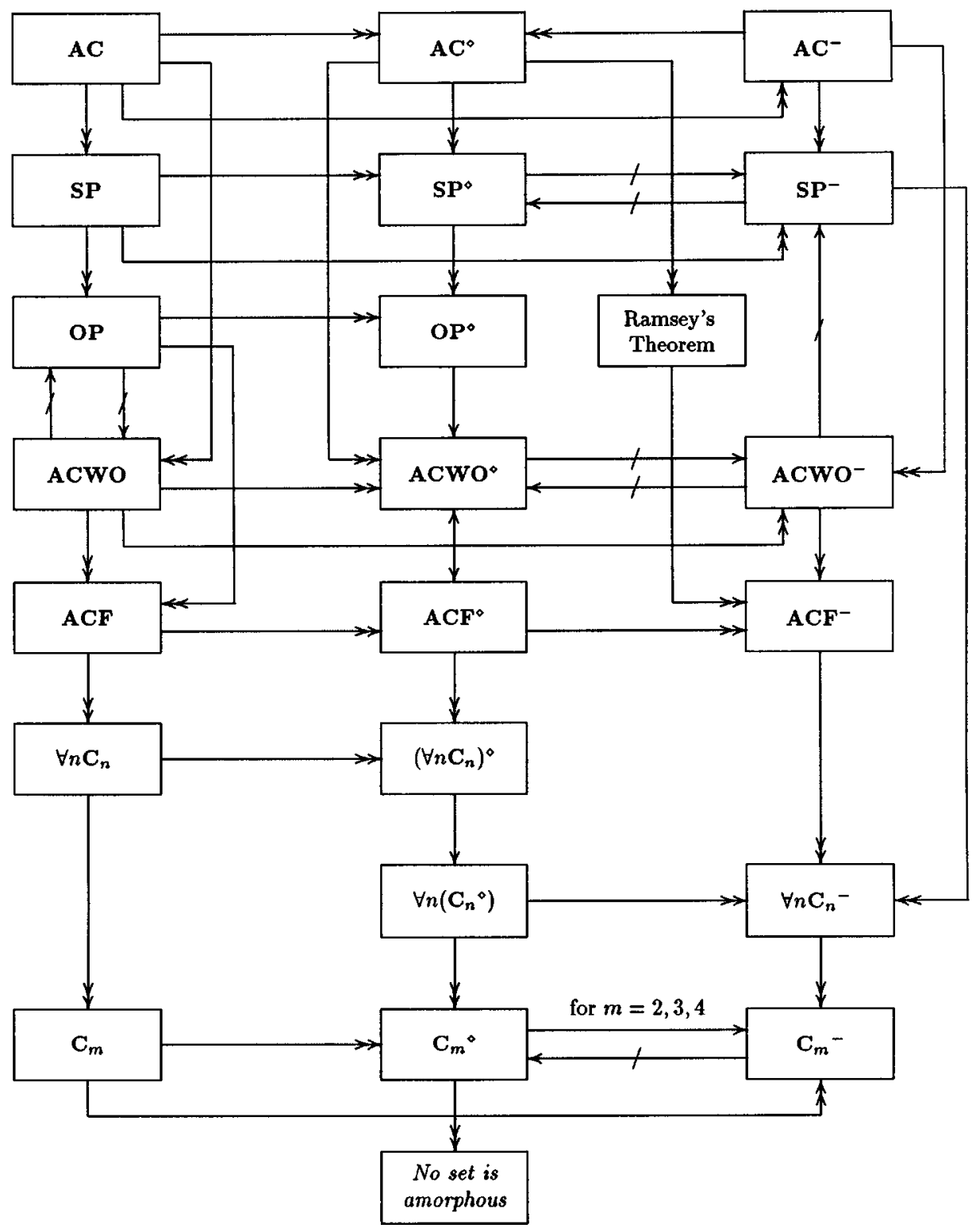

Proposition 3.2. ACWO $^{-}$holds in the Basic Fraenkel Model.

Proof. We show first a general fact; in BFM, if $Z$ is well orderable and $H \subset A$ is a support for $Z$, then $H$ is actually a supersupport for $Z$.

Assume $H$ is a support for the well orderable set $Z$, and that there is $z \in Z$ not supported by $H$. Just as in the lemma of [Bl, p. 389], there is an infinite subset of $Z$ which is in one-one correspondence with an infinite subset of $A$. This would imply that this infinite subset of $A$ is well orderable and therefore, that it contains a countable infinite subset. This is a contradiction since countable sets are not amorphous. In conclusion $H$ is a supersupport for $Z$.

Let $F$ be an infinite collection of well orderable sets. 
CASE 1: $F$ is well orderable.

Fix a support $E$ for $F$. By the fact above, it is actually a supersupport for $F$ and also for each of its elements.

Take a choice function $g$ for $F$ (in the base model, which satisfies ZFC); $E$ is a support for this choice function, since, if $\pi \in \operatorname{fix}(E)$, then for every pair $\left(X_{n}, g\left(X_{n}\right)\right) \in g$ we have:

$$
\begin{aligned}
\pi\left(X_{n}, g\left(X_{n}\right)\right) & =\left(\pi X_{n}, \pi\left(g\left(X_{n}\right)\right)\right) \\
& =\left(X_{n}, g\left(X_{n}\right)\right)
\end{aligned}
$$

because $\pi \in \operatorname{fix}(E), E$ is a supersupport for $X_{n}$ and $g\left(X_{n}\right) \in X_{n}$.

(Note that this proof actually shows that in the Basic Fraenkel Model every well orderable family of well orderable sets has a choice function.)

CAsE 2: $F$ is not well orderable.

By the lemma of [Bl, p. 389], there is $X_{0} \in F$, an atom $a$, and a finite set of atoms $E$ such that $a \notin E$ and

$$
f=\left\{\left(\pi a, \pi X_{0}\right): \pi \in \operatorname{fix}(E)\right\}
$$

is a bijection from $A \backslash(E)$ onto an infinite subset $F^{\prime} \subset F$. Pick $x_{0} \in X_{0}$ and define $g$ by

$$
g=\left\{\left(\pi X_{0}, \pi x_{0}\right): \pi \in \operatorname{fix}(E)\right\}
$$

$g$ is in the model since it is supported by $E$. To show that $g$ is a function notice that $X_{0}$ is supported by $E \cup\{a\}$ since for $\pi \in \operatorname{fix}(E \cup\{a\}), \pi a=a$ and therefore $X_{0}=f(a)=f(\pi a)=\pi X_{0}$. By the fact at the beginning of the proof, $E \cup\{a\}$ supports $x_{0}$ as well. If $\pi_{1}, \pi_{2} \in \operatorname{fix}(E)$ and $\pi_{1} X_{0}=\pi_{2} X_{0}$, then, since $f$ is a bijection, $\pi_{2}^{-1} \pi_{1} a=a$, and thus $\pi_{1} X_{0}=\pi_{2} X_{0}$.

The domain of $g$ is the range of $f$, an infinite subset of $F$, and obviously, $g$ is a choice function.

\section{Proposition 3.3. $\mathbf{S P}^{-}$holds in the Basic Fraenkel Model.}

Proof. We will show that in BFM, for every infinite family $F$ of sets there is an infinite subfamily for which there is a function selecting proper subsets.

CAsE 1: $F$ is well orderable.

In this case there is a supersupport $E$ for $F$. Fix a well order of $F$, say $F=$ $\left\{X_{\xi}: \xi \in \delta\right\}$.

a) If $E$ is also a supersupport for infinitely many of the sets in $F$, then any selector function (which exists in the base model by AC) will also be supported by $E$, and thus belongs to the permutation model.

b) If for infinitely many $\xi$ 's the set $\left\{x \in X_{\xi}: x\right.$ is supported by $\left.E\right\}$ is a proper, non-empty subset of $X_{\xi}$, then this is the selector function. It is in the permutation model since it is supported by $E$.

c) For all $\xi \in \delta$ (except, possibly, a finite number), every $x \in X_{\xi}$ is moved by some $\pi \in \operatorname{fix}(E)$.

Claim: In this case there must exist an atom $a \in A$ such that for infinitely many $\xi$ 's, $E \cup\{a\}$ supports a proper non-empty subset of $X_{\xi}$.

Otherwise, if for every $a, E \cup\{a\}$ only supports proper non-empty subsets of finitely many of the $X_{n}$ 's, we could define $f: A \rightarrow \omega$ by $f(a)=$ maximum $n$ for 
which $E \cup\{a\}$ supports a proper non-empty subset of $X_{n}$, (and $f(a)=0$ if no such maximum $n$ exists).

The function $f$ is in the model since it is supported by $E$. We show that the function $f$ has unbounded image. Fix $n \in \omega$, and for every $y \in X_{n}$, let $E_{y}$ be the minimal support for $y$. If $\bigcup\left\{E_{y}: y \in X_{n}\right\}$ is finite, then it is a supersupport for $X_{n}$, and therefore $X_{n}$ is well orderable and $E$, being support for it, is a supersupport, and a supersupport for any of its subsets. In this case, for every $a \in A$, we have $f(a) \geq n$. If $\bigcup\left\{E_{y}: y \in X_{n}\right\}$ is infinite, then there are $y, z \in X_{n}$, and $a \in A$ such that $a \in E_{y} \backslash E_{z}$. The set $\left\{y \in X_{n}: a \in E_{y}\right\}$ is supported by $E \cup\{a\}$, and therefore $f(a) \geq n$. This gives us a way to write $A$ as an increasing union of sets (increasing properly infinitely many times). This contradicts that $A$ is amorphous, which proves the claim.

Given an atom $a$ as in the claim, the function selecting from $X_{n}$ a proper nonempty subset supported by $E \cup\{a\}$ from each $X_{\xi}$ selects a proper subset from infinitely many of the $X_{\xi}$ 's. This function is in the model since it is supported by $E \cup\{a\}$.

CASE 2: $F$ is not well orderable.

Proceeding as in the previous theorem, choose $X_{0} \in F$, an atom $a$, and a finite set of atoms $E$ such that $a \notin E$ and $f=\left\{\left(\pi a, \pi X_{0}\right): \pi \in f i x(E)\right\}$ is a bijection from $A \backslash(E)$ onto an infinite subset $F^{\prime} \subset F$. If $X_{0}$ is well orderable, proceed as in Case 2 of Proposition 3.2. Otherwise, $\exists x_{0} \in X_{0}$ and an atom $b$ which is in the minimal support of $x_{0}$ but not in $E \cup\{a\}$. For $\pi X_{0} \in F^{\prime}$ define

$$
G\left(\pi X_{0}\right)=\left\{y \in \pi X_{0}: b \text { is in the minimal support of } y\right\} .
$$

A modified version of the Basic Fraenkel Model allows us to show that $\mathbf{A C W O}^{-}$ does not imply $\mathbf{S P}^{-}$. Namely, organize the atoms in a countable sequence $\left\{A_{n}\right.$ : $n \in \omega\}$ of infinite sets, and consider the group generated by the permutations of each $A_{n}$, with finite supports.

Proposition 3.4. ACWO ${ }^{\diamond}$ is false in the Basic Fraenkel Model.

Proof. Clearly ACWO $\mathbf{A C}^{\diamond}$ implies $\mathbf{C}_{2} \diamond$, and by the results of [ $\left.\mathrm{Tr}\right]$ mentioned above, this implies that there are no amorphous sets. Since $A$ is amorphous, we get the desired result.

Proposition 3.5. $\mathbf{A C}^{-}$is false in $\mathrm{BFM}$.

Proof. By part 1 of 1.2 and the fact that $\mathbf{A} \mathbf{C}^{\diamond}$ does not hold in BFM.

In the cylinder model, $\mathbf{A C W O}^{\diamond}$ holds but $\mathbf{A C W O}^{-}$does not.

For the Selection Principle none of the implications hold. The cylinder model provides a model for $\mathbf{S P}^{\diamond}$ and $\neg \mathbf{S} \mathbf{P}^{-}$, and BFM satisfies $\mathbf{S P}^{-}$and $\neg \mathbf{S} \mathbf{P}^{\diamond}$.

\section{Ramsey's Theorem, partitions And Weak Choice PRINCIPLeS}

Ramsey's Theorem [Ra] follows from $\mathbf{A C}^{\diamond}$ and it implies $\mathbf{A C F}^{-}$[K12]. We explore the relationship of Ramsey's Theorem with other weak choice principles.

A consequence of Lemma 2.2 is that Ramsey's Theorem holds in CM, but $\mathbf{A C W O}^{-}$does not hold there. On the other hand, from $\mathbf{S P}^{\diamond}$ (which is, in our 
scheme, the next weaker principle after $\mathbf{A C}^{\diamond}$ ), Ramsey's Theorem is not provable, as can be seen observing Cohen's basic symmetric model (see below).

If an infinite set is not amorphous, we say it is partible (it can be split into two infinite subsets) [G].

In $[G]$, Gonzalez analyses a series of properties arising from the possibility of splitting infinite sets. We have already mentioned that "every infinite set can be split in two infinite subsets" is not provable in ZF (it is false in BFM and this can be transferred to a model of ZF).

A set is $\aleph_{0}$-partible if it can be split into a countable sequence of subsets.

Ramsey's Theorem does not imply $\mathbf{C}_{2}$, nor does it imply "every set is partible". This can be shown observing that in the Basic Fraenkel Model Ramsey's Theorem holds [Bl], but $\mathbf{C}_{2}$ is false, and the set of atoms is not partible.

In the basic Cohen model, the selection principle holds (and therefore so does $\mathbf{S P}^{\diamond}$ and $\mathbf{S P}^{-}$), but Ramsey's Theorem does not ([Kl1], [Bl]). Thus, a consequence of the following lemma is that the principle "Every set is $\aleph_{0}$-partible" does not imply Ramsey's Theorem.

The principle ACWO holds also in the basic Cohen model (together with its reduced and diminished versions), so none of them implies Ramsey's Theorem.

Lemma 4.1. The Selection Principle implies every infinite set is $\aleph_{0}$-partible.

Proof. Given an infinite set $X$, let $f: P_{\geq 2}(X) \rightarrow P(X)$ be a selection function (i.e. for every $S \subseteq X$ with at least two elements, $\phi \subsetneq f(S) \subsetneq S)$.

From $f$ we will obtain an $\omega$-sequence $A_{0}, A_{1}, \ldots$ of distinct subsets of $X$, from which it follows that $X$ is $\aleph_{0}$-partible.

If $X-f(X)$ is infinite, put $A_{0}=f(X)$, otherwise $A_{0}=X-f(X)$. If we have defined $A_{n}$, let $B_{n}=\bigcup_{i=0}^{n} A_{i}$ and put $A_{n+1}=f\left(X-B_{n}\right)$ if $X-f\left(X-B_{n}\right)$ is infinite; otherwise, put $A_{n+1}=X_{f}\left(X-B_{n}\right)$.

Corollary 4.2. "Every infinite set is $\aleph_{0}$-partible" does not imply Ramsey's Theorem.

\section{REFERENCES}

[Bl] Blass, A., Ramsey's Theorem in the hierarchy of choice principles, Journal of Symbolic Logic 42 (1977), 367-390. MR 57:5751

[Br] Brunner N., Sequential compactness and the axiom of choice, Notre Dame Journal of Formal Logic 24 (1983), 89-92. MR 84f:04009

[G] Gonzalez, C., Partitioning an Infinite Set, Preprint (1994).

[Je1] Jech, T., The Axiom of Choice, North Holland, 1973. MR 53:139

[Je2] Jech, T., Set Theory, Academic Press, 1978. MR 80:03062

[Kl1] Kleinberg, E. M., The independence of Ramsey's Theorem, Journal of Symbolic Logic 34 (1969), 205-206. MR 41:51

[K12] , Infinitary Combinatorics, Cambridge Summer School in Mathematical Logic (A.R.D. Mathias, Ed.), Lecture Notes in Math., vol. 337, Springer Verlag, 1973, pp. 361418. MR 49:2400

[Mo] Montenegro, C., Personal communication.

[Pi] Pincus, D., Individuals in Zermelo-Fraenkel Set Theory, Doctoral Dissertation, Harvard University, 1969.

[Pi1]_ Zermelo-Fraenkel Consistency Results by Fraenkel-Mostowski Methods, Journal of Symbolic Logic 37 (1972), 721-724. MR 49:2374

[Pi2] - Adding dependent choice, Annals of Mathematical Logic 11 (1977), 105-145. MR 56:11791 
[Ra] Ramsey F. P., On a problem of formal logic, Proc. of the London Mathematical Society, Ser, 2 30, Part 4 (1928), 338-384.

[Tr] Truss, J., Classes of Dedekind finite cardinals, Fundamenta Mathematicae 84 (1974), 187-208. MR 57:9541

Department of Mathematics, Instituto Venezolano de Investigaciones Científicas, Apartado 21827, Caracas 1020-A, Venezuela

Current address, Omar De la Cruz: Department of Mathematics, University of Florida, Gainesville, Florida 32611

E-mail address: odlc@math.ufl.edu

E-mail address: cdiprisc@ivic.ivic.ve 